\title{
Delineation of clinical target volume for postoperative radiotherapy in stage IIIA-pN2 non-small-cell lung cancer
}

This article was published in the following Dove Press journal:

OncoTargets and Therapy

19 February 2016

Number of times this article has been viewed

\author{
Xuquan Jing ${ }^{1,2, *}$ \\ Xue Meng ${ }^{2, *}$ \\ Xindong Sun ${ }^{2}$ \\ Jinming $\mathrm{Yu}^{2}$
}

'School of Medicine and Life Sciences, University of Jinan-Shandong Academy of Medical Sciences, ${ }^{2}$ Department of Radiation Oncology, Shandong Cancer Hospital and Institute, Jinan, People's Republic of China

*These authors contributed equally to this work
Correspondence: Jinming Yu Department of Radiation Oncology, Shandong Cancer Hospital and Institute, No 440 Jiyan Road, Jinan, Shandong Province, 250I I7, People's Republic of China

Tel +8653187984729

Fax +86 53। 87984079

Email sdyujinming@I26.com
Abstract: With the high locoregional relapse rate and the improvement of radiation technology, postoperative radiotherapy (PORT) has been widely used in the treatment of completely resected stage IIIA-pN2 non-small-cell lung cancer (NSCLC). However, there is still no definitive consensus on clinical target volume for the $\mathrm{pN} 2$ subgroup. This review will discuss how to delineate the clinical target volume (CTV) for pN2 subgroups of IIIA-N2 NSCLC based on the published literature and to investigate the optimal PORT CTV in this cohort of patients. Besides overall survival (OS), locoregional recurrence (LR), and radiotherapy-related toxicity of this subset of the population in the modern PORT era, selection of proper patients will also be considered in this review. In summary, it is appropriate to include involved lymph node stations and uninvolved stations at high risk in PORT CTV for patients with pN2 disease when PORT is administered. PORT can reduce LR and has the potential to improve OS. In the current era of modern radiation technology, PORT can be administered safely with well-tolerated toxicity. Clinicopathological characteristics may be helpful in selecting proper candidates for PORT.

Keywords: stage IIIA, non-small-cell lung carcinoma, postoperative radiotherapy, target volume

\section{Introduction}

Completely resected stage IIIA-N2 non-small-cell lung cancer (NSCLC), which is defined as ipsilateral, mediastinal, or subcarinal lymph node involvement, is considered to be a heterogeneous subgroup of diseases, with 5-year overall survival (OS) rates in the range of $7 \%-34 \% .{ }^{1-3}$ The advantages of postoperative adjuvant chemotherapy (POCT) have been confirmed, and it is now considered to be the standard treatment. ${ }^{4-7}$ However, locoregional recurrence (LR) as high as $40 \%$ has been reported, even after radical resection followed by adjuvant chemotherapy. ${ }^{4-7}$ Considering the high rate of LR, the use of postoperative radiotherapy (PORT) has been considered for the multidisciplinary treatment of patients with $\mathrm{N} 2$ disease, ${ }^{2}$ in order to improve locoregional control (LRC) and further increase OS.

Patients with stage IIIA-N2 NSCLC show substantial heterogeneity in clinical presentation, treatment, and prognosis. Accordingly, with the aim of generating rational treatment guidelines, IIIA-N2 NSCLC has been classified into four subsets (Table 1), which have been published previously. ${ }^{8}$ Of note, for patients with N2 NSCLC identified preoperatively $\left(\mathrm{IIIA}_{3}\right.$ ), either definitive chemoradiation therapy or induction therapy followed by surgery is recommended. In addition, in patients with NSCLC who have bulky N2 disease (IIIA 4 ) and good performance status, combination platinum-based chemotherapy and concomitant radiotherapy are recommended. ${ }^{9}$ Then, stage IIIA-pN2 
Table I Subsets of stage IIIA-N2

\begin{tabular}{ll}
\hline Subset & Description \\
\hline IIIA & Incidental nodal metastases found on final pathology examination of the resection specimen \\
$\mathrm{IIIA}_{2}$ & Nodal (single-station) metastases recognized intraoperatively \\
$\mathrm{IIIA}_{3}$ & Nodal metastases (single or multiple station) recognized by prethoracotomy staging (mediastinoscopy, other nodal biopsy, or PET scan) \\
$\mathrm{IIIA}_{4}$ & Bulky or fixed multistation N2 disease \\
\hline
\end{tabular}

Abbreviations: PET, positron emission tomography; N2, ipsilateral mediastinal or subcarinal lymph node involvement.

patients who are defined as only IIIA 1 and IIIA 2 after fully resected surgery and adjuvant chemotherapy are suitable to receive PORT.

However, there is still no definite agreement on the extent of target delineation of the PORT clinical target volume (CTV) for the treatment of completely resected IIIA-pN2 NSCLC patients because of the paucity of evidence of contouring the target field. Among 17 thoracic radiation oncologists invited to contour routine CTV for representative patients, large variability has been observed in PORT field design. ${ }^{10}$ A Japanese survey confirmed the existence of variations in the practice of contouring the CTV as well. ${ }^{3}$ Fortunately, these variances will be largely reduced if delineation guidelines are followed. ${ }^{10,11}$ In the context of the ongoing debate over the delineation of the CTV, we summarize, in this review, the available data on volume design, discussing the following questions: Which regions have been considered to be at high risk of failure for PORT CTV? What reasons may influence the determination of the design of target? How to deliver PORT safely, and what is the effect of PORT? And who are the more suitable patients that would benefit most from PORT?

\section{Regions with high risk of failure as PORT CTV}

PORT is an accepted avenue for treating NSCLC patients after complete resection to reduce locoregional failure. With the advent of modern radiotherapy, a crucial question has arisen regarding the design of PORT treatment fields in this setting, which traditionally included the entire mediastinum. Limited volumes may improve the therapeutic ratio of PORT with a reduction in treatment-associated morbidity. Anatomical and patterns-of-failure studies provide guidance to the more intelligent design of PORT fields based on the most likely sites of locoregional failure. ${ }^{12,13}$ This approach will facilitate the use of smaller radiotherapy fields with correspondingly less treatment-related morbidity. Accordingly, using these anatomical and clinical data, one can create "more customized" PORT fields. ${ }^{14}$

Anatomical studies concerning lymphatic mapping show direct drainage from pulmonary segments into mediastinal lymph nodes, bypassing intrapulmonary and hilar lymph nodes. ${ }^{14}$ Furthermore, since PORT is a prophylactic-intent treatment, locoregional patterns of failure depend mainly on the location and number of resected lymph nodes during surgery. It is therefore necessary to define how to deal with the mediastinal nodes in operation. Following the results of a randomized trial, systematic mediastinal lymph node sampling is equivalent to complete lymphadenectomy with regard to survival for NSCLC disease. ${ }^{15,16}$ Thus, either of them could be used when delivering complete resection. Explicitly, an adequate mediastinal lymphadenectomy should include right upper paratracheal stations (lymph nodes stations [LNS], LNS 2R), right lower paratracheal stations (LNS 4R), subcarinal stations (LNS 7), paraesophageal stations (LNS 8), and pulmonary ligament stations (LNS 9) for right-sided cancers; and left upper paratracheal stations (LNS 4L), subaortic stations (LNS 5), para-aortic stations (LNS 6), LNS 7, LNS 8, and LNS 9 should be sampled for left-sided cancers. ${ }^{17}$ It has been recommended that if sampling is taken, the minimum requirements include the removal of at least six lymph nodes from hilar and mediastinal stations, at least one of which must be subcarinal. ${ }^{18}$ The lymph node map from the International Association for the Study of Lung Cancer (IASLC) may be helpful for surgery. ${ }^{19}$

Subsequent analysis is an attempt to obtain more available data concerning locoregional nodal failure patterns in the subgroup of pN2 patients, and to determine the optimal PORT CTV in this cohort of patients.

\section{Areas with consistently high failure rate form PORT CTV with basic consensus}

Even in patients who received complete resection, failures of bronchial stump (BS) and lymph nodes were observed in clinical practice. Under these circumstances, thereby, regions including $\mathrm{BS}$ and mediastinal lymph nodes will be investigated sequentially to better determine the PORT CTV. Comprehensively based on surgical data and regularity of lymphatic drainage, elementary agreement on CTV is as follows.

For all patients enrolled in the study of Kelsey et al, ${ }^{20}$ the most common site of failure was the BS region, accounting 
for $44 \%$. This result was similar to that of another trial in which the rate of BS failure was $41 \% .{ }^{21}$ Subset analysis showed that both left-sided tumors and right-located tumors had a high recurrence rate in the BS region. Given the historical results showing that the BS region had a higher rate of recurrence after surgery, encompassing the BS in the CTV is recommended. As for regions with metastases verified in the pathological examination, there is no doubt that those involved LNS should be included in the PORT CTV because of the highest risk of recurrence. ${ }^{11}$

The principal drainage of the lung is generally through the ipsilateral hilar nodes (LNS10R or 10L) and LNS $7 .{ }^{22}$ The ipsilateral hilar is, therefore, more apt to be recurrent. In former trials, the rate of failure of the ipsilateral hilar region was, respectively, $15 \%, 19 \%$, and $22 \%$, which supported the aforementioned view. ${ }^{20,21,23}$ When the probability of recurrent risk of involvement for lymph node is $>10 \%$, the corresponding site should be included in the CTV in light of the very high rate of recurrence. ${ }^{24}$ Concordantly, high recurrence of hilar nodes is supported by the previously published data..$^{20,21,23}$ Therefore, in consideration of the consistently high rate of recurrence, the ipsilateral hilar node is recommended for inclusion in the CTV regardless of the laterality of lung tumors. Moreover, the recurrence rate of LNS 7 was reported to be as high as $37 \%$ in a study that examined the patterns of locoregional failure in completely resected stage IIIA-N2 NSCLC cases. ${ }^{25}$ In three published trials, all specialists recommended LNS 7 for the postoperative target volume without hesitation for $\mathrm{pN} 2$ NSCLC patients. ${ }^{10,25,26}$

The right lung drains principally through the right mediastinum, whereas the left lung drains the lymph through both sides of the mediastinum. As a result of different regularity of drainage, we thus discuss the recurrence rate of followed lymph nodes according to the laterality of the primary tumor. Table 2 shows published data that we have recalculated. For the right-sided tumors, LNS $4 \mathrm{R}$ have a high failure rate. The recurrent rate varied from $19 \%$ to $26 \%$, significantly higher than the benchmark $(10 \%)$. With regard to the left-sided lung tumors, LNS $4 \mathrm{~L}$ was found to have a high rate $(14 \%)$ in only one study, but less than $5 \%$ in the other two trials. And if the characteristics of drainage are considered, including LNS 4L in the CTV for left tumors is not paradoxical. Besides, LNS 5 had a relatively high rate recurrence, ranging from $8 \%$ to $16 \%$.

As for patients who did not receive radiotherapy to the supraclavicular region during primary treatment, the recurrence rate in this area was not higher than $10 \% .{ }^{27}$ The irradiation of these lymphatic areas could improve local control, but the improvement for OS remains unclear. Consequently, it is believed that the supraclavicular areas should not be included in the CTV for adjuvant treatment with curative purpose. ${ }^{24}$

Based on available information about patterns of local relapse after surgery as well as studies related to the PORT CTV for completely resected N2 NSCLC patients, ${ }^{10,11,25,26}$ the BS, any involved LNS, LNS 10R or 10L, LNS 7, and LNS 4R or $4 \mathrm{~L}$ are recommended for inclusion in the CTV of PORT for $\mathrm{pN} 2$ disease. In the case of left-sided tumors, LNS 5 should also be encompassed in the CTV. Moreover, the supraclavicular areas should not be included in the CTV.

\section{Areas with inconsistent probability of failure as controversial PORT CTV}

Although a consistently high probability of failure sites has been identified, there exist many arguments for CTV. The main reason for the controversy in regard to designing CTV is the incongruent rate of failure for given lymph nodes. For the right lung carcinoma, LNS $2 \mathrm{R}$ also had a high rate of failure. In one trial, the recurrent rate is as high as $26 \%,{ }^{25}$ even higher than that of LNS 4R reported in other studies. So Feng et $\mathrm{al}^{25}$ recommend LNS $2 \mathrm{R}$ for inclusion in PORT CTV.${ }^{16}$ Concerning the strikingly high rate of recurrence in this region, including this area in the CTV may be appropriate. With respect to LNS 3, in the Kelsey et a ${ }^{20}$ study, a failure rate of close to $10 \%$ was observed, which may to some

Table 2 The rate of local recurrence (\%)

\begin{tabular}{|c|c|c|c|c|c|c|c|c|c|c|c|}
\hline \multirow[t]{2}{*}{ Study } & \multirow[t]{2}{*}{ Primary site } & \multirow[t]{2}{*}{ Ipsilateral hilum } & \multicolumn{9}{|c|}{ Location of lymph nodes } \\
\hline & & & $2 \mathbf{L}$ & $2 \mathbf{R}$ & 3 & 4L & $4 R$ & 5 & 6 & 7 & 8 \\
\hline \multirow[t]{2}{*}{ Kelsey et $\mathrm{al}^{20}$} & $R L$ & 6.9 & 1.3 & 12.5 & 5.5 & 4.2 & 23.6 & 4.2 & 5.5 & 13.9 & 1.3 \\
\hline & LL & 16. I & $\mathrm{I} .8$ & 3.6 & 7.1 & 3.6 & 14.3 & 16.1 & 1.8 & 14.3 & 0 \\
\hline \multirow[t]{2}{*}{ Qin et $\mathrm{al}^{21}$} & $\mathrm{RL}$ & 8.9 & 0.6 & 15.6 & 3.9 & 3.3 & 26.1 & I.I & 0 & 17.2 & 2.8 \\
\hline & LL & 11.6 & 1.9 & 2.9 & 9.7 & 4.8 & 16.5 & 7.8 & 3.9 & 19.4 & 1 \\
\hline \multirow[t]{2}{*}{ Feng et $\mathrm{a}^{25}$} & $\mathrm{RL}$ & 19 & NA & 26 & NA & NA & 19 & NA & NA & 15 & NA \\
\hline & LL & 12 & NA & NA & NA & 14 & 22 & 12 & 12 & 20 & NA \\
\hline
\end{tabular}

Note: The rate in Table 2 is the number of each metastasis lymph node divided by the total number of recurrences in the left/right lung.

Abbreviations: RL, right lung; LL, left lung; NA, not available; $2 \mathrm{~L}$, left upper paratracheal stations; $2 \mathrm{R}$, right upper paratracheal stations; 3 , prevascular and prevertebral stations; 4L, left lower paratracheal stations; 4R, right lower paratracheal stations; 5, subaortic stations; 6, para-aortic stations; 7, subcarinal stations; 8, paraesophageal stations. 
degree explain why a few radiation oncologists suggest that LNS 3A up to the top of the aortic arch is supposed to be included in the PORT CTV for $\mathrm{pN} 2$ disease. The accurate recurrence of this area is also unsettled. Although Kepka et $\mathrm{al}^{11}$ advised that PORT CTV should include LNS 3A up to the top of the aortic arch, practically, the coverage of this node may still lead to some clinical uncertainties.

With regard to the left-located tumors, the condition of LNS 6 is similar to that of LNS 4L. More than 10\% occurred only in one trial for LNS $6 .{ }^{25}$ In Spoelstra et al's study, ${ }^{10}$ LNS 6 were included in the CTV, which is parallel to the findings of Feng, ${ }^{25}$ who suggested that LNS 6 are apt to be recurrent even after radical resection. However, LNS 6 were not included in the CTV in a prospective trial conducted by Kepka et al. ${ }^{11}$ Unfortunately, Kepka et al ${ }^{11}$ did not point out the recurrent rate of LNS 6. These results contribute to the uncertainty of including these areas. Including it in CTV may reduce local relapse or, in contrast, increase radiotherapyinduced toxicity. Perhaps further research could solve the dilemma. What is notable here is that LNS 4R had a higher failure rate than LNS 4L for the left lung (Table 2), 22\% at the highest. Our suggestion is that except for LNS 4L, LNS $4 \mathrm{R}$ should also be included in the CTV for left primary tumor to reduce the high failure rate.

In the study of Spoelstra et al, ${ }^{10}$ with any possible extension to the mediastinal pleura adjacent to the resected tumor bed, all the lymph nodes that lie between two noncontiguous nodal stations that have contained metastases at any stage were also included in the CTV. When metastases are labeled in a nodal station, the next nodal station both superior and inferior to it should also be included in the CTV. However, if this protocol were adopted in clinical practice, the volume delineated for the CTV could become extremely large in some cases. For example, in the case of LNS 7 involved, LNS 8 should theoretically be included, with the effect that the lower limit will be at the gastroesophageal junction. However, as for LNS 8 and its lower limit, in the study of Feng et al, ${ }^{25}$ no locoregional failure at LNS 8 or 9 was observed in any of the patients, including those with lower lobe tumors. Increasing the target volume to include the lower zone would result in a significant increase in radiation volume. ${ }^{12}$ It is not known with certainty whether the inclusion of the lower zone is necessary.

In conclusion, based on comprehensive evidence concerning mediastinal lymphatic drainage and the distribution of nodal involvement, it is appropriate to treat involved lymph node stations and uninvolved stations as being at high risk for patients with $\mathrm{pN} 2$ disease when PORT is administered.

\section{Potential explanations of the argument of PORT CTV}

The chief reason for controversial target design is the inconsistent risk of failure presented earlier. Furthermore, the different rates of recurrence may be explained by the following causes. Patients may receive different surgical procedures such as lobectomy or pneumonectomy; even these operations can have the effect of radical resection. Providing the same surgical process, the surgical centers may show variability in dealing with complete operation, and different surgeons have divergent variations. The station and the number of resected lymph nodes are not concordant and are important factors influencing the prognosis.

The histology of resected tissues may also influence locoregional failure (LRF) as adenocarcinoma and squamous cell carcinoma as well as other subtypes are heterogeneous disease. The relationship between the target volume design and the pathology depends on the state of primary tumors and lymph nodes. For primary tumors, when the CTV is created by the addition of margin to the gross tumor volume (GTV), a margin of 8 and $6 \mathrm{~mm}$ is needed, respectively, for adenocarcinoma (ADC) and squamous cell carcinoma (SCC) for covering $95 \%$ of the microscopic extension. ${ }^{28}$ However, this review focuses on the population who have received complete resection. The primary tumors no longer bias the target design. With regard to lymph nodes, one study shows that the incidence of nodal extracapsular extension between groups divided by histological type does not reach statistical significance. ${ }^{29}$ This study also demonstrates that the margin of $3 \mathrm{~mm}$ for lymph nodes was determined for lymph nodes of sizes of up to $20 \mathrm{~mm}$. For lymph nodes of $21-30 \mathrm{~mm}$, the extracapsular microscopic extension might have reached up to $12 \mathrm{~mm}$. Unfortunately, for patients with full resection of lymph nodes, this result is not completely suitable for prophylactic irradiation. In summary, there are no studies published focusing on the correlation between the PORT CTV and pathology for patients who received complete resection. The delineation of CTV is still considered to be more of an art than a science.

In addition to the various rates of relapse, certain other aspects should also be contemplated. The degree of differentiation may influence radio-oncologists contouring the PORT CTV. Patients with poor differentiation are more likely to be recurrent; the CTV herein would be expanded naturally or half unconsciously with experts being more cautious. However, the studies related to CTV delineation did not adequately discuss this section. ${ }^{10,11,20,25}$ Besides, age and the pulmonary function are crucial factors. When patients are relatively older and have poor pulmonary function, in 
contrast to the poor differentiation, specialists would decrease the PORT CTV in order to minimize radiotherapy toxicity or even stop those patients receiving radiotherapy, because these patients have bad tolerance for radiotherapy. Described in the study of Feng et al, ${ }^{25}$ the proposed CTV for left lung cancers includes the BS and LNSs 2R, 2L, 4R, 4L, 5, 6, 7, and 10 to $11 \mathrm{~L}$; and the proposed CTV for right lung cancers includes the BS and LNSs 2R, 4R, 7, and 10 to 11R. Comparing left lung cancer with right lung cancer, the PORT CTV for the whole group of right lung did not include LNS 5 and LNS 6. How does the radio-oncologist contour the PORT CTV if patients have characteristics of poor differentiation, younger age, and good tolerance? Perhaps including LNS 5 and LNS 6 in the CTV may be suitable. It is necessary that we tackle these individuals to be individualized or personalized basis.

Furthermore, the data used in proposing CTV are retrospective. Given the nature of retrospective analysis, there exist limitations such as unknown selection bias. Based on retrospective data on recurrence, the proposed CTV may differ from each other, ${ }^{10,11,20,25}$ which is the common failing of former studies. Because the delineation of target volume for PORT is based upon lower-level evidence, some deviations come from experience. In the absence of a golden standard for delineating CTV, differences exist between delineators.

In studies discussed previously, nodal failures were defined as a new or enlarging lymph node $\geq 1 \mathrm{~cm}$ in the short axis on CT or hypermetabolic on positron-emission tomography, regardless of size. Pathological verification of recurrence was not mandatory. Pathological confirmation of recurrent disease was obtained only in about $24 \%-60 \% .^{20,25}$ Most patients were presumed to have recurrence based only on radiographic studies that have a finite positive predictive value. There are methodological problems in detecting the specific location of recurrence based on radiographic studies. So the locations of true recurrence may be various and influence the decision for CTV.

PORT is generally administered sequentially following the completion of adjuvant chemotherapy for patients who have received full resection. ${ }^{30}$ The relationship between previous chemotherapy regimen and target volume design was not illuminated in published studies. Of note, when concurrent chemoradiotherapy is delivered for unresectable disease, clinically significant radiation toxicity including esophagitis and pneumonitis was more frequent in the gemcitabine arm, according to the results of both the CALGB 30105 trial and the KASLC 0401 trial. ${ }^{31,32}$ In clinical practice, however, we do not observe that chemotherapy increases the treatment-related toxicity for subsequent radiotherapy.
Therefore, previous chemotherapy could be let alone, or a regimen with low toxicity for adjuvant chemotherapy could be chosen to guarantee safety of treatment.

\section{The implementation and role of PORT in pN2 disease Dose prescription and normal tissue constraint}

When delivering radiotherapy postoperatively, margins, doses, technology used, and organs at risk should be carefully considered. Margins added to the CTV to create the planning target volume (PTV) in NSCLC have two main ingredients: one for motion (mainly respiratory, but also cardiac beats) of tumor and organs at risk, and another for setup errors. It is common in clinical practice to add the margin directly to the CTV without separating the two components. Generally, a $0.5-1 \mathrm{~cm}$ margin is added to the CTV to create the PTV to minimize the uncertainties of respiratory motion and setup. ${ }^{11,33}$ Respiratory tracking or gating systems or fourdimensional tomography (4D-CT) scanning, particularly 4D-CT planning, may allow for decreased PTV margins. ${ }^{34}$

When administering PORT, radiation therapy doses after complete resection tend to be at 50-54 Gy in 1.8-2 Gy fractions in most series. ${ }^{35,36} 50$ Gy (2 Gy per fraction, total 25 fractions) have been used in the ongoing clinical trial registered on the Clinical Trials website http://clinicaltrials.gov under number NCT00880971. ${ }^{37}$ Higher doses have historically been avoided because of the higher risk of postoperative complications. ${ }^{38}$ Encouraging results for optimal radiotherapy were revealed by recent studies with different fraction schedules or radiation dose escalation. A phase III trial (CHART) indicated that continuous, hyperfractionated, accelerated radiotherapy compared with standard fractionation may improve survival in NSCLC patients. ${ }^{39}$ The CHARTWEL trial could not corroborate these results but demonstrated a profound tendency for a decrease in LR. ${ }^{40}$

Modern conformal radiotherapy, such as three-dimensional conformal radiotherapy (3D-CRT) and intensity-modulated radiotherapy (IMRT) should be used in order to minimize unnecessary normal tissue irradiation, reduce toxicity, and improve outcome. ${ }^{41}$ Furthermore, another paramount factor in planning PORT radiotherapy is protection of critical normal lung and heart tissue from excessive irradiation. It is essential in this setting to accurately delineate both critical normal structures and target volume. 3D-CRT with detailed attention to dose-volume histograms (DVH) is pivotal to the safe planning and delivery of PORT. The target volume should generally not be irradiated $>54 \mathrm{~Gy}$, and the lung V20 (volume \% of lung receiving $\geq 20$ Gy) must be minimized, preferably 
to $<25 \%$. In addition, a mean lung dose of $<20 \mathrm{~Gy}$, mean esophagus dose of $<34 \mathrm{~Gy}$, maximum esophagus dose of $<80 \mathrm{~Gy}$, and less than $50 \%$ of the heart receiving 40 Gy (V40 <50\%) were required. ${ }^{42}$

\section{Survival and safety of PORT}

Although earlier randomized trials were unable to demonstrate a survival benefit associated with PORT in completely resected $\mathrm{N} 2$ patients, ${ }^{43,44}$ with the rapidly advancing radiotherapy techniques and the new enthusiasm for PORT, a growing number of studies including meta-analyses and trials have proven that PORT is associated with better survival and local control. ${ }^{25,33,45-49}$ The subgroup analyses of PORT meta-analysis and its update revealed that for patients with N2 involvement, there was neither harm nor benefit from PORT concerning survival, ${ }^{43,50}$ although they have been criticized for their drawbacks. ${ }^{51,52}$ On the contrary, another meta-analysis, published in 2013, affirmed that modern PORT with linear accelerators could increase the absolute 5-year OS by $13 \%$ in stage IIIA-N2 NSCLC patients. ${ }^{45}$ The favorable impact of PORT on OS in N2 subgroup was in agreement with the findings of more recent studies, such as Surveillance, Epidemiology, and End Results (SEER) and an Adjuvant Navelbine International Trialist Association (ANITA) trial. ${ }^{7,46,53}$ Furthermore, the results of an analysis of the National Cancer Data Base revealed that PORT was associated with better survival for patients with pN2 nodes. ${ }^{54}$ Even after adjuvant chemotherapy, modern PORT seems to confer an additional OS advantage. ${ }^{55}$ What is more, several large retrospective studies have provided evidence for the advantage of PORT in N2 disease. . $^{33,47-49}$

Although the impact of PORT on OS is still unclear, there exists circumstantial evidence suggesting that LRC could be improved in stage IIIA-N2 disease. In a modern PORT meta-analysis, ${ }^{45}$ the authors concluded that locoregional relapse rate (LRR) significantly decreased with PORT using either beam quality. The results of that meta-analysis have shown that modern PORT with linear accelerators reduces LRR from $30 \%$ to $10 \%$ with RR of 0.31 . As part of a SEER study, 5-year univariate and multivariate analyses confirmed that PORT reduced LR in N2 patients. ${ }^{46}$ The lower rate of LR with PORT was in line with the outcome of an ANITA trial. ${ }^{53}$ A retrospective study of 221 cases predicted that the LRR was $30.2 \%$ and $39.2 \%$ in the PORT and non-PORT arms, respectively, which led to a significantly longer locoregional recurrence-free survival duration in favor of PORT $\left(\chi^{2}, 5.048 ; P=0.025\right) .{ }^{48}$ Parallel results concerning LR have been confirmed in substantial retrospective studies. . $33,47,48,56,57^{2}$ In brief, all those findings discussed earlier offer convincing evidence of LRC benefit with PORT applied to patients with resected N2 NSCLC.

With the improvement in radiotherapy techniques, the rate of radiation-related toxicity has not been higher in quite a few studies after the wide use of modern PORT techniques. ${ }^{26,35,44,58-60}$ A retrospective study concentrating on the issue of toxicity, led by Machtay, found that the 4-year actuarial rate of death from intercurrent disease (DID) was $13.5 \%$, implying a slight but statistically insignificant difference compared with the expected rate of intercurrent death for a matched population. ${ }^{35}$ An analogous study, the ECOG3590, has yielded almost identical results. ${ }^{44}$ In addition, the results of a SEER data-based study have proven that the risk of heart disease mortality connected with PORT has lessened in more recent years because of the improvements in treatment planning and in the modern delivery of radiotherapy. A nonrandomized trial suggested that PORT with contemporary techniques did not increase cardiopulmonary morbidity and did not impair pulmonary function tests results and quality of life in the evaluation performed 2 years after treatment. This technique for PORT was shown to be safe, revealing no signs of late toxicity. ${ }^{26}$ Furthermore, the reduction in PORT-related morbidity in consequence of development in technology is borne out by several other studies. ${ }^{23,47,48,59,61}$ All of these findings might support the notion that there has been no significant increase in death resulting from the use of PORT in recent times and that modern radiation techniques have reduced the severe toxicity of PORT.

\section{Selection of proper candidates for PORT}

A feasible and reasonable way to improve the outcome of PORT is to select proper candidates or the high subgroup that can derive greater benefit from PORT among the N2 population. A recently published retrospective study showed that PORT significantly improved the OS only in patients with SI (smoking index: number of cigarettes smoked per day $\times$ number of cigarette-years) $\leq 400$, cN2, pT3, SCC (squamous cell carcinoma), or LNM (metastatic lymph nodes) $\geq 4 .{ }^{62}$ Among the five factors, each factor was equally given one score. Patients with 1-5 of all the factors were scored from 0-5. The authors established a predictive model of evaluating the necessity of PORT. The patients were then divided into low PORT index (PORT-I) group (score 0-1), middle PORT-I group (score 2), and high PORT-I group (score 3-5). Cox regression implied that PORT did not improve the OS of patients in the low PORT-I group (hazard ratio, HR $=0.988$, $P=0.984$ ), tended to improve the OS in the middle PORT-I 
group $(\mathrm{HR}=0.614, P=0.179)$, and significantly improved the OS in the high PORT-I group ( $\mathrm{HR}=0.371, P=0.001$ ). The conclusions of that investigation were that PORT was strongly recommended for patients with three or more of the five factors of SI $\leq 400, \mathrm{cN} 2$, pT3, SCC, and $\geq 4$ positive nodes, and that the prediction model was valuable in selecting patients with resected IIIA-N2 NSCLC who are fit for PORT.

A noteworthy fact is that smoking is one of the most significant poor prognostic factors of NSCLC, ${ }^{63,64}$ including resected NSCLC. ${ }^{65}$ Current smokers still have a poorer locoregional control after PORT for NSCLC. ${ }^{66}$ However, that PORT significantly improved the OS of patients with SI $\leq 400$ was shown in the aforementioned study, and may be explained by the fewer comorbidities, better tolerance, and tumor response to PORT in non- or light smokers than in heavy smokers. Further studies herein are warranted to identify the biological basis for the effect of smoking on radiotherapy in NSCLC patients. Although SCC was associated with lower OS compared with adenocarcinoma,${ }^{67}$ the prognostic significance of SCC is still controversial in fully resected pN2 NSCLC. ${ }^{48}$

Furthermore, a retrospective study has revealed that PORT leads to longer OS times for patients with resected $\mathrm{pN} 2$ NSCLC with a negative extracapsular extension (ECE) status but not with a positive ECE status. ${ }^{68}$ Furthermore, another retrospective study conducted by Matsuguma et $\mathrm{al}^{69}$ suggested that PORT for radical resection N2 NSCLC patients was more effective for multiple-station mediastinal lymph nodes metastases than single-station metastasis. These results are in close agreement with some other works. ${ }^{23,56}$ Wisnivesky et al ${ }^{70}$ found that PORT is not associated with improved survival in elderly (age $\geq 65$ years) patients with stage III NSCLC who have N2 disease and undergo surgical resection; meanwhile, these data suggested that PORT should not be used routinely to treat these patients outside research trials.

Patients with "inclusion criteria" of selection may benefit much more from PORT, thanks to these available studies. A definitive conclusion still needs further randomized controlled trials.

\section{Conclusion and future directions}

Treating only involved lymph node stations and uninvolved stations at high risk will minimize incidental radiation to surrounding normal structures and, consequently, minimize treatment-related mortality. However, delineation of radiation field has so far failed to reach full agreement, and it should be comprehensively based on the surgical situation and primary tumor and risk of relapse to be individualized and normalized. In clinical practice, the PORT CTV for completely resected N2
NSCLC could consult the published literature discussed earlier. Considering comprehensively available data, it is appropriate to treat involved lymph node stations and uninvolved stations at high risk for patients with $\mathrm{pN} 2$ disease when PORT is administered. Therefore, the BS region, any involved LNS, ipsilateral hilar nodes, ipsilateral lower paratracheal nodes, and subcarinal nodes should at least be included in the PORT CTV regardless of the location of primary tumors. As for the left-located tumors, LNS 5 should also be included in the CTV. It is generally acknowledged that PORT could reduce the risk of locoregional failure considerably; however, the impact of PORT in OS is still being hotly debated. With the advent of modern radiation techniques and equipment, adjuvant radiotherapy after operation can be delivered safely for completely resected N2 NSCLC patients with an acceptable treatmentrelated toxicity in the light of existing evidence. Individualized strategies based on different clinical-pathological features might be considered. High-level evidence to guide the optimal utilization of PORT for N2 NSCLC patients is still insufficient, warranting further studies to obtain additional information to identify the optimal PORT CTV for pN2 NSCLC patients.

\section{Acknowledgments}

This review is supported by the National Natural Science Foundation of China (81472810), the special fund for Scientific Research in Public Interest (201402011), and the Science and Technology Development Plans of Shandong Province (2014GSF118138).

\section{Disclosure}

The authors report no conflicts of interest in this work.

\section{References}

1. Andre F, Grunenwald D, Pignon JP, et al. Survival of patients with resected N2 non-small-cell lung cancer: evidence for a subclassification and implications. J Clin Oncol. 2000;18(16):2981-2989.

2. Gopal RS, Dubey S, Rosenzweig KE, et al. ACR Appropriateness Criteria $^{\circledR}$ on induction and adjuvant therapy for stage N2 non-small-cell lung cancer: expert panel on radiation oncology-lung. Int J Radiat Oncol Biol Phys. 2010;78(4):969-974.

3. Uno T, Sumi M, Kihara A, et al. Postoperative radiotherapy for non-smallcell lung cancer: results of the 1999-2001 patterns of care study nationwide process survey in Japan. Lung Cancer. 2007;56(3):357-362.

4. Winton T, Livingston R, Johnson D, et al. Vinorelbine plus cisplatin vs. observation in resected non-small-cell lung cancer. $N$ Engl J Med. 2005; 352(25):2589-2597.

5. Arriagada R, Bergman B, Dunant A, Le Chevalier T, Pignon JP, Vansteenkiste J. Cisplatin-based adjuvant chemotherapy in patients with completely resected non-small-cell lung cancer. NEnglJMed.2004;350(4): 351-360.

6. Arriagada R, Auperin A, Burdett S, et al. Adjuvant chemotherapy, with or without postoperative radiotherapy, in operable non-small-cell lung cancer: two meta-analyses of individual patient data. Lancet. 2010 375(9722):1267-1277. 
7. Douillard JY, Rosell R, De Lena M, et al. Adjuvant vinorelbine plus cisplatin versus observation in patients with completely resected stage IB-IIIA non-small-cell lung cancer (Adjuvant Navelbine International Trialist Association [ANITA]): a randomised controlled trial. Lancet Oncol. 2006;7(9):719-727.

8. Ruckdeschel JC. Combined modality therapy of non-small cell lung cancer. Semin Oncol. 1997;24(4):429-439.

9. Ramnath N, Dilling TJ, Harris LJ, et al. Treatment of stage III non-small cell lung cancer: diagnosis and management of lung cancer, 3rd ed: American College of Chest Physicians evidence-based clinical practice guidelines. Chest. 2013;143(Suppl 5):e314S-e340S.

10. Spoelstra FO, Senan S, Le Pechoux C, et al. Variations in target volume definition for postoperative radiotherapy in stage III non-small-cell lung cancer: analysis of an international contouring study. Int J Radiat Oncol Biol Phys. 2010;76(4):1106-1113.

11. Kepka L, Bujko K, Bujko M, et al. Target volume for postoperative radiotherapy in non-small cell lung cancer: results from a prospective trial. Radiother Oncol. 2013;108(1):61-65.

12. Miles EF, Kelsey CR, Kirkpatrick JP, Marks LB. Estimating the magnitude and field-size dependence of radiotherapy-induced mortality and tumor control after postoperative radiotherapy for non-small-cell lung cancer: calculations from clinical trials. Int J Radiat Oncol Biol Phys. 2007;68(4):1047-1052.

13. Kelsey CR, Clough RW, Marks LB. Local recurrence following initial resection of NSCLC: salvage is possible with radiation therapy. CancerJ. 2006;12(4):283-288.

14. Saynak M, Higginson DS, Morris DE, Marks LB. Current status of postoperative radiation for non-small-cell lung cancer. Semin Radiat Oncol. 2010;20(3):192-200.

15. Darling GE, Allen MS, Decker PA, et al. Randomized trial of mediastinal lymph node sampling versus complete lymphadenectomy during pulmonary resection in the patient with N0 or N1 (less than hilar) non-small cell carcinoma: results of the American College of Surgery Oncology Group Z0030 Trial. J Thorac Cardiovasc Surg. 2011;141(3):662-670.

16. Allen MS, Darling GE, Pechet TT, et al. Morbidity and mortality of major pulmonary resections in patients with early-stage lung cancer: initial results of the randomized, prospective ACOSOG Z0030 trial. Ann Thorac Surg. 2006;81(3):1013-1019; discussion 1019-1020.

17. Darling GE, Allen MS, Decker PA, et al. Number of lymph nodes harvested from a mediastinal lymphadenectomy: results of the randomized, prospective American College of Surgeons Oncology Group Z0030 trial. Chest. 2011;139(5):1124-1129.

18. De Leyn P, Lardinois D, Van Schil P, et al. European trends in preoperative and intraoperative nodal staging: ESTS guidelines. $J$ Thorac Oncol. 2007;2(4):357-361.

19. Rusch VW, Asamura H, Watanabe H, Giroux DJ, Rami-Porta R, Goldstraw P. The IASLC lung cancer staging project: a proposal for a new international lymph node map in the forthcoming seventh edition of the TNM classification for lung cancer. J Thorac Oncol. 2009;4(5):568-577.

20. Kelsey CR, Light KL, Marks LB. Patterns of failure after resection of nonsmall-cell lung cancer: implications for postoperative radiation therapy volumes. Int J Radiat Oncol Biol Phys. 2006;65(4):1097-1105.

21. Qin P, Yuan Z, Wang J, et al. [Research on Postoperative Radiotherapy for Non-small Cell Lung Cancer of Stage IIIA (N2) according to the Failure Patterns after Pulmonary Resection]. Zhongguo Fei Ai Za Zhi. 2009;12(10):1095-1100. Chinese.

22. Naruke T, Suemasu K, Ishikawa S. Lymph node mapping and curability at various levels of metastasis in resected lung cancer. $J$ Thorac Cardiovasc Surg. 1978;76(6):832-839.

23. Ichinose $Y$, Kato H, Koike $T$, et al. Overall survival and local recurrence of 406 completely resected stage IIIa-N2 non-small cell lung cancer patients: questionnaire survey of the Japan Clinical Oncology Group to plan for clinical trials. Lung Cancer. 2001;34(1):29-36.

24. Kiricuta IC. Selection and delineation of lymph node target volume for lung cancer conformal radiotherapy. Proposal for standardizing terminology based on surgical experience. Strahlenther Onkol. 2001; $177(8): 410-423$.
25. Feng W, Fu XL, Cai XW, et al. Patterns of local-regional failure in completely resected stage IIIA(N2) non-small cell lung cancer cases: implications for postoperative radiation therapy clinical target volume design. Int J Radiat Oncol Biol Phys. 2014;88(5):1100-1107.

26. Kepka L, Bujko K, Orlowski TM, et al. Cardiopulmonary morbidity and quality of life in non-small cell lung cancer patients treated with or without postoperative radiotherapy. Radiother Oncol. 2011;98(2): 238-243.

27. Kiricuta IC, Mueller G, Stiess J, Bohndorf W. The lymphatic pathways of non-small cell lung cancer and their implication in curative irradiation treatment. Lung Cancer. 1994;11(1-2):71-82.

28. Giraud P, Antoine M, Larrouy A, et al. Evaluation of microscopic tumor extension in non-small-cell lung cancer for three-dimensional conformal radiotherapy planning. Int J Radiat Oncol Biol Phys. 2000; 48(4):1015-1024.

29. Yuan S, Meng X, Yu J, et al. Determining optimal clinical target volume margins on the basis of microscopic extracapsular extension of metastatic nodes in patients with non-small-cell lung cancer. Int J Radiat Oncol Biol Phys. 2007;67(3):727-734.

30. Willers H, Stinchcombe TE, Barriger RB, et al. ACR Appropriateness Criteria $\left({ }^{\circledR}\right)$ induction and adjuvant therapy for N2 non-small-cell lung cancer. Am J Clin Oncol. 2015;38(2):197-205.

31. Socinski MA, Blackstock AW, Bogart JA, et al. Randomized phase II trial of induction chemotherapy followed by concurrent chemotherapy and dose-escalated thoracic conformal radiotherapy (74 Gy) in stage III non-small-cell lung cancer: CALGB 30105. J Clin Oncol. 2008; 26(15):2457-2463.

32. Oh IJ, Kim KS, Kim YC, et al. A phase III concurrent chemoradiotherapy trial with cisplatin and paclitaxel or docetaxel or gemcitabine in unresectable non-small cell lung cancer: KASLC 0401. Cancer Chemother Pharmacol. 2013;72(6):1247-1254.

33. Scotti V, Meattini I, Saieva C, et al. Post-operative radiotherapy in N2 non-small cell lung cancer: a retrospective analysis of 175 patients. Radiother Oncol. 2010;96(1):84-88.

34. Slotman BJ, Lagerwaard FJ, Senan S. 4D imaging for target definition in stereotactic radiotherapy for lung cancer. Acta Oncol. 2006;45(7):966-972.

35. Machtay M, Lee JH, Shrager JB, Kaiser LR, Glatstein E. Risk of death from intercurrent disease is not excessively increased by modern postoperative radiotherapy for high-risk resected non-small-cell lung carcinoma. J Clin Oncol. 2001;19(19):3912-3917.

36. Krupitskaya Y, Loo BW Jr. Post-operative radiation therapy (PORT) in completely resected non-small-cell lung cancer. Curr Treat Options Oncol. 2008;9(4-6):343-356.

37. Chinese Academy of Medical Sciences. To Evaluate Postoperative 3D Conform Radiotherapy in Patients With IIIA (N2) Non-small Cell Lung Cancer (NCT00880971). Available from: https://clinicaltrials. gov/ct $2 /$ show/NCT00880971? term $=$ To + Evaluate + Postoperative +3 D+Conform+Radiotherapy+in+Patients+With+IIIA+\%28N2\%29+ Non-small+Cell+Lung+Cancer.\&rank=1. NLM identifier: NCT00880971. Accessed January 26, 2016.

38. Sonett JR, Suntharalingam M, Edelman MJ, et al. Pulmonary resection after curative intent radiotherapy ( $>59 \mathrm{~Gy})$ and concurrent chemotherapy in non-small-cell lung cancer. Ann Thorac Surg. 2004;78(4): 1200-1205; discussion 1206.

39. Soliman M, Yaromina A, Appold S, et al. GTV differentially impacts locoregional control of non-small cell lung cancer (NSCLC) after different fractionation schedules: subgroup analysis of the prospective randomized CHARTWEL trial. Radiother Oncol. 2013;106(3): 299-304.

40. Baumann M, Herrmann T, Koch R, et al. Final results of the randomized phase III CHARTWEL-trial (ARO 97-1) comparing hyperfractionatedaccelerated versus conventionally fractionated radiotherapy in non-small cell lung cancer (NSCLC). Radiother Oncol. 2011;100(1):76-85.

41. De Ruysscher D, Faivre-Finn C, Nestle U, et al. European Organisation for Research and Treatment of Cancer recommendations for planning and delivery of high-dose, high-precision radiotherapy for lung cancer. J Clin Oncol. 2010;28(36):5301-5310. 
42. Jackson A, Marks LB, Bentzen SM, et al. The lessons of QUANTEC: recommendations for reporting and gathering data on dose-volume dependencies of treatment outcome. Int J Radiat Oncol Biol Phys. 2010; 76(Suppl 3):S155-S160.

43. Stewart L, Parmar M, Souhami R, et al. Postoperative radiotherapy in non-small-cell lung cancer: systematic review and meta-analysis of individual patient data from nine randomised controlled trials. PORT Meta-analysis Trialists Group. Lancet. 1998;352(9124):257-263.

44. Wakelee HA, Stephenson P, Keller SM, et al. Post-operative radiotherapy (PORT) or chemoradiotherapy (CPORT) following resection of stages II and IIIA non-small cell lung cancer (NSCLC) does not increase the expected risk of death from intercurrent disease (DID) in Eastern Cooperative Oncology Group (ECOG) trial E3590. Lung Cancer. 2005;48(3):389-397.

45. Billiet C, Decaluwe H, Peeters S, et al. Modern post-operative radiotherapy for stage III non-small cell lung cancer may improve local control and survival: a meta-analysis. Radiother Oncol. 2014;110(1):3-8.

46. Lally BE, Zelterman D, Colasanto JM, Haffty BG, Detterbeck FC, Wilson LD. Postoperative radiotherapy for stage II or III non-smallcell lung cancer using the surveillance, epidemiology, and end results database. J Clin Oncol. 2006;24(19):2998-3006.

47. Zou B, Xu Y, Li T, et al. A multicenter retrospective analysis of survival outcome following postoperative chemoradiotherapy in non-small-cell lung cancer patients with N2 nodal disease. Int J Radiat Oncol Biol Phys. 2010;77(2):321-328.

48. Dai H, Hui Z, Ji W, et al. Postoperative radiotherapy for resected pathological stage IIIA-N2 non-small cell lung cancer: a retrospective study of 221 cases from a single institution. Oncologist. 2011;16(5):641-650.

49. Mantovani C, Levra NG, Filippi AR, et al. Postoperative radiotherapy for patients with completely resected pathologic n2 non-small-cell lung cancer: a retrospective analysis. Clin Lung Cancer. 2013;14(2): 194-199.

50. PORT Meta-analysis Trialists Group. Postoperative radiotherapy for non-small cell lung cancer. Cochrane Database Syst Rev. 2005;(2) CD002142.

51. Bogart JA, Aronowitz JN. Localized non-small cell lung cancer: adjuvant radiotherapy in the era of effective systemic therapy. Clin Cancer Res. 2005;11(13 Pt 2):5004s-5010s.

52. Bonner JA, Tincher SA, Fiveash JB. Balancing the possible effectiveness of postoperative radiotherapy for non-small-cell lung cancer against the possible detriment of radiation-induced toxicity. J Clin Oncol. 2001; 19(19):3905-3907.

53. Douillard JY, Rosell R, De Lena M, Riggi M, Hurteloup P, Mahe MA. Impact of postoperative radiation therapy on survival in patients with complete resection and stage I, II, or IIIA non-small-cell lung cancer treated with adjuvant chemotherapy: the adjuvant Navelbine International Trialist Association (ANITA) randomized trial. Int J Radiat Oncol Biol Phys. 2008;72(3):695-701.

54. Mikell JL, Gillespie TW, Hall WA, et al. Post-Operative Radiotherapy (PORT) is associated with better survival in non-small cell lung cancer with involved N2 lymph nodes: results of an analysis of the National Cancer Data Base. J Thorac Oncol. 2015;10(3):462-471.

55. Robinson CG, Patel AP, Bradley JD, et al. Postoperative radiotherapy for pathologic n2 non-small-cell lung cancer treated with adjuvant chemotherapy: a review of the national cancer data base. J Clin Oncol. 2015;33(8):870-876.

OncoTargets and Therapy

\section{Publish your work in this journal}

OncoTargets and Therapy is an international, peer-reviewed, open access journal focusing on the pathological basis of all cancers, potential targets for therapy and treatment protocols employed to improve the management of cancer patients. The journal also focuses on the impact of management programs and new therapeutic agents and protocols on
56. Kim BH, Kim HJ, Wu HG, et al. Role of postoperative radiotherapy after curative resection and adjuvant chemotherapy for patients with pathological stage N2 non-small-cell lung cancer: a propensity score matching analysis. Clin Lung Cancer. 2014;15(5):356-364.

57. Bradley JD, Paulus R, Graham MV, et al. Phase II trial of postoperative adjuvant paclitaxel/carboplatin and thoracic radiotherapy in resected stage II and IIIA non-small-cell lung cancer: promising long-term results of the Radiation Therapy Oncology Group - RTOG 9705. J Clin Oncol. 2005;23(15):3480-3487.

58. Bradley J, Graham MV, Winter K, et al. Toxicity and outcome results of RTOG 9311: a phase I-II dose-escalation study using three-dimensional conformal radiotherapy in patients with inoperable non-small-cell lung carcinoma. Int J Radiat Oncol Biol Phys. 2005;61(2):318-328.

59. Keller SM, Adak S, Wagner $\mathrm{H}$, et al. A randomized trial of postoperative adjuvant therapy in patients with completely resected stage II or IIIA non-small-cell lung cancer. Eastern Cooperative Oncology Group. N Engl J Med. 2000;343(17):1217-1222.

60. Lally BE, Detterbeck FC, Geiger AM, et al. The risk of death from heart disease in patients with nonsmall cell lung cancer who receive postoperative radiotherapy: analysis of the surveillance, epidemiology, and end results database. Cancer. 2007;110(4):911-917.

61. von Lieven H, Burkhardt E. Postoperative radiotherapy of NSCLC outcome after 3-D treatment planning. Strahlenther Onkol. 2001;177(6): 302-306.

62. Hui Z, Dai H, Liang J, et al. Selection of proper candidates with resected pathological stage IIIA-N2 non-small cell lung cancer for postoperative radiotherapy. Thorac Cancer. 2015;6(3):346-353.

63. Ferketich AK, Niland JC, Mamet R, et al. Smoking status and survival in the national comprehensive cancer network non-small cell lung cancer cohort. Cancer. 2013;119(4):847-853.

64. Kogure Y, Ando M, Saka H, et al. Histology and smoking status predict survival of patients with advanced non-small-cell lung cancer. Results of West Japan Oncology Group (WJOG) Study 3906L. J Thorac Oncol. 2013;8(6):753-758.

65. Hanagiri T, Sugio K, Mizukami M, et al. Significance of smoking as a postoperative prognostic factor in patients with non-small cell lung cancer. J Thorac Oncol. 2008;3(10):1127-1132.

66. Nguyen SK, Masson-Cote L, Fortin A, Dagnault A. Influence of smoking status on treatment outcomes after post-operative radiation therapy for non-small-cell lung cancer. Radiother Oncol. 2010;96(1):89-93.

67. Asamura H, Goya T, Koshiishi Y, et al. A Japanese Lung Cancer Registry study: prognosis of 13,010 resected lung cancers. $J$ Thorac Oncol. 2008;3(1):46-52.

68. Moretti L, Yu DS, Chen H, et al. Prognostic factors for resected nonsmall cell lung cancer with pN2 status: implications for use of postoperative radiotherapy. Oncologist. 2009;14(11):1106-1115.

69. Matsuguma H, Nakahara R, Ishikawa Y, et al. Postoperative radiotherapy for patients with completely resected pathological stage IIIA-N2 non-small cell lung cancer: focusing on an effect of the number of mediastinal lymph node stations involved. Interact Cardiovasc Thorac Surg. 2008;7(4):573-577.

70. Wisnivesky JP, Halm EA, Bonomi M, Smith C, Mhango G, Bagiella E. Postoperative radiotherapy for elderly patients with stage III lung cancer. Cancer. 2012;118(18):4478-4485.

patient perspectives such as quality of life, adherence and satisfaction The manuscript management system is completely online and includes a very quick and fair peer-review system, which is all easy to use. Visit http://www.dovepress.com/testimonials.php to read real quotes from published authors. 